\title{
Cross-reactivity of each fraction among cereals in children with wheat allergy
}

\author{
Mari Takei ${ }^{1}$, Akemi Saito ${ }^{1}$, Noriyuki Yanagida ${ }^{2}$, Sakura Sato $^{3}$, and Motohiro Ebisawa ${ }^{1}$ \\ ${ }^{1}$ National Hospital Organization, Sagamihara National Hospital \\ ${ }^{2}$ National Hospital Organization Sagamihara National Hospital \\ ${ }^{3}$ Sagamihara National Hospital
}

December 27, 2021

\begin{abstract}
Background: Cross-reactivity between wheat and other cereals is an essential issue in the management of wheat allergy. Few studies have reported in vitro cross-reactivity in immediate-type wheat allergy. This study aimed to examine cross-reactivity of the three fractions (albumin/globulin, gliadin, and glutenin fractions) among cereals in children with wheat allergy. Methods: Sera from 128 children with immediate-type wheat allergy were collected. We measured specific immunoglobulin E (sIgE) levels against each fraction of wheat, barley, and rye by using an enzyme-linked immunosorbent assay (ELISA). Cross-reactivities of each fraction among wheat, barley, and rye were examined via inhibition ELISA. Results: All subjects were sensitized to all the fractions of wheat, and also those of barley and rye. The wheat sIgE levels were significantly higher than those of barley and rye in all the fractions ( $\mathrm{p}$ [?] 0.001) and were significantly correlated with sIgE levels to them in each fraction ( $\mathrm{r}=$ 0.887-0.969, $\mathrm{p}<0.001)$. On inhibition ELISA, wheat inhibited the IgE binding to most of the solid phases at the lower protein levels compared to barley and rye in all fractions. Conclusions: In children with immediate-type wheat allergy, sensitization to all the three fractions of wheat was observed. In addition, they showed sensitization to barley and rye caused by in vitro cross-reactivity with wheat in each fraction. When managing children with wheat allergy, sensitization to barley and rye caused by the cross-reactivities should be considered.
\end{abstract}

\section{Hosted file}

Main_Document_211216.docx available at https://authorea.com/users/436775/articles/550777cross-reactivity-of-each-fraction-among-cereals-in-children-with-wheat-allergy 


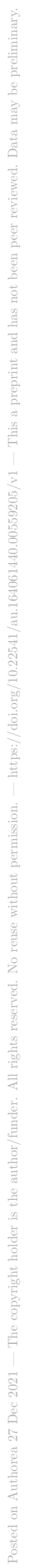
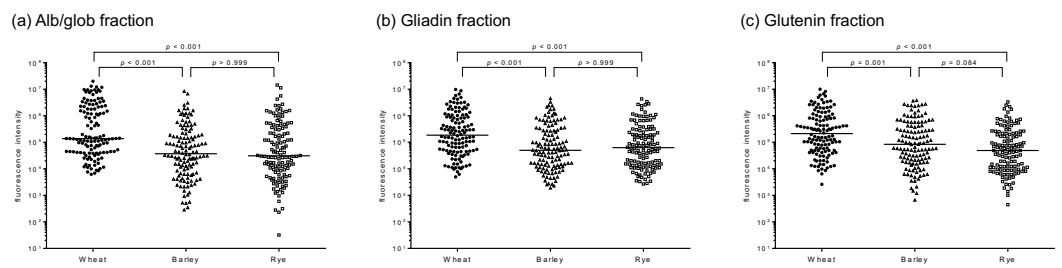

2 
(a) Alb/glob fraction
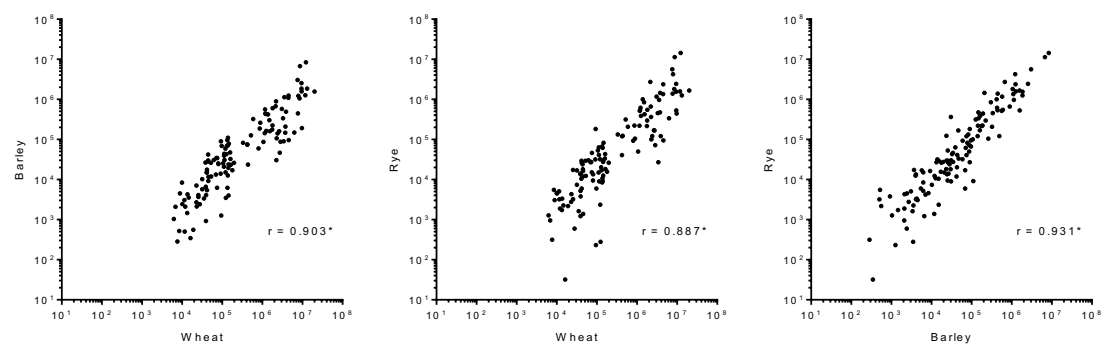

(b) Gliadin fraction
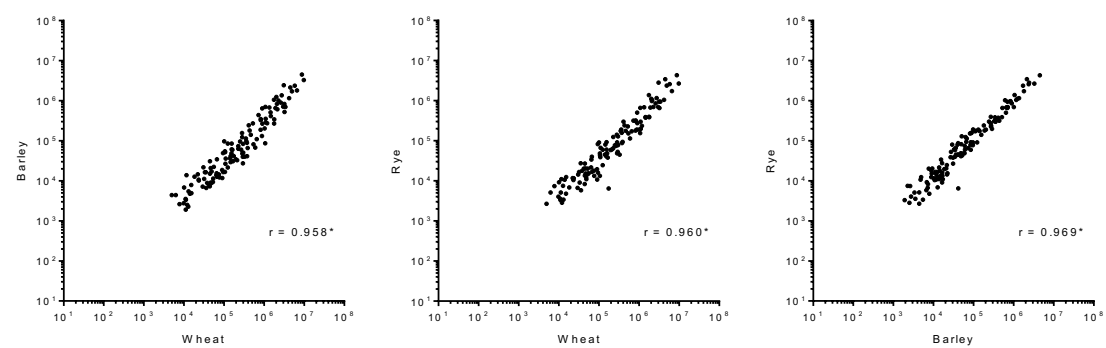

(c) Glutenin fraction
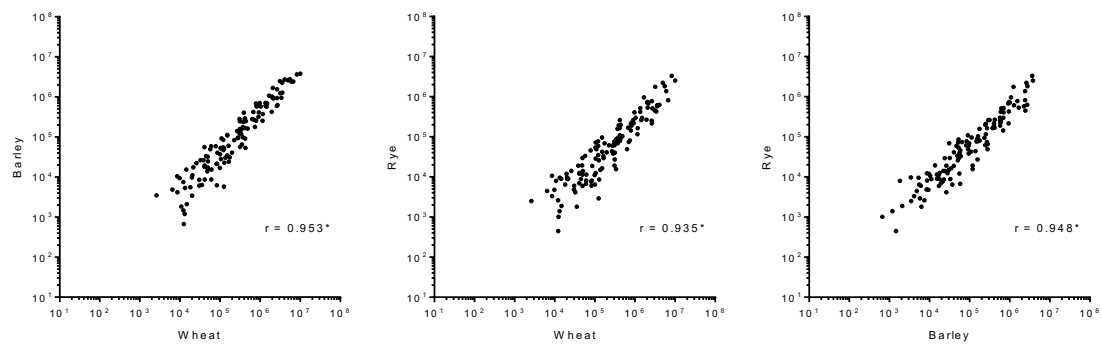
Alb/glob fraction

(a) Wheat

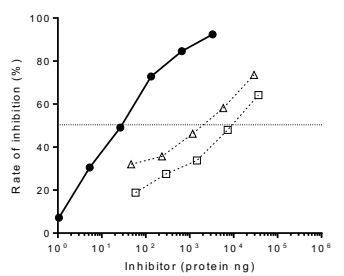

Gliadin fraction

(d) Wheat

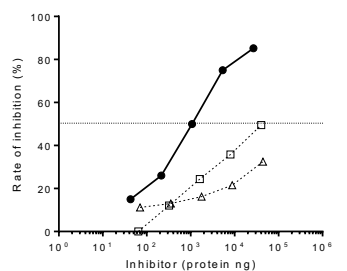

Glutenin fraction

(g) Wheat

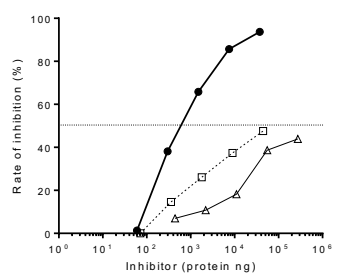

(b) Barley

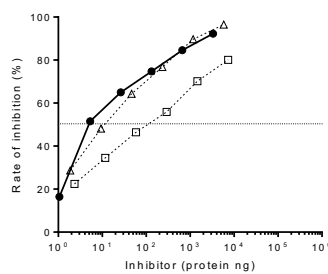

(e) Barley

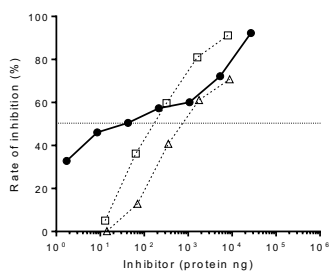

(h) Barley

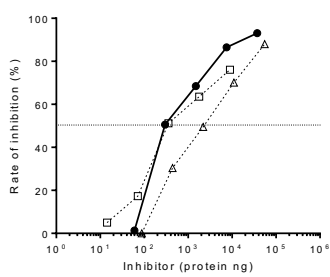

(c) Rye

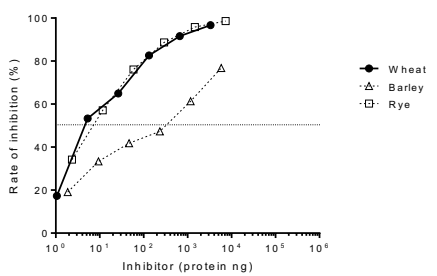

(f) Rye

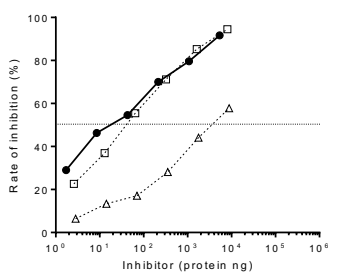

- Whenter

(i) Rye

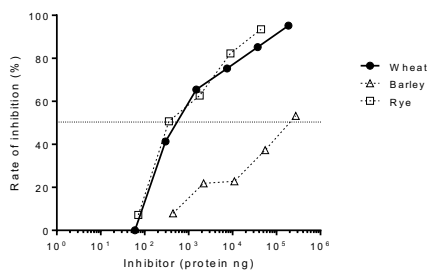


(a)

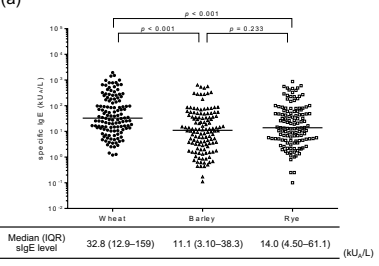

(b)

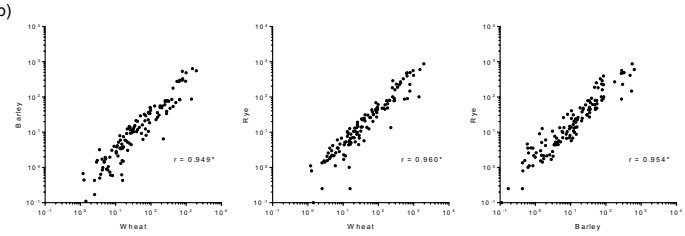

